\title{
Smart Demand for Frequency Regulation: Experimental Results
}

\author{
Douglass, Philip James; Garcia-Valle, Rodrigo; Nyeng, Preben; Østergaard, Jacob; Togeby, Mikael
}

\section{Published in:}

IEEE Transactions on Smart Grid

Link to article, DOI:

10.1109/TSG.2013.2259510

Publication date:

2013

Link back to DTU Orbit

Citation $(A P A)$ :

Douglass, P. J., Garcia-Valle, R., Nyeng, P., Østergaard, J., \& Togeby, M. (2013). Smart Demand for Frequency Regulation: Experimental Results. IEEE Transactions on Smart Grid, 4(3), 1713 - 1720 . https://doi.org/10.1109/TSG.2013.2259510

\section{General rights}

Copyright and moral rights for the publications made accessible in the public portal are retained by the authors and/or other copyright owners and it is a condition of accessing publications that users recognise and abide by the legal requirements associated with these rights.

- Users may download and print one copy of any publication from the public portal for the purpose of private study or research.

- You may not further distribute the material or use it for any profit-making activity or commercial gain

- You may freely distribute the URL identifying the publication in the public portal 


\title{
Smart Demand for Frequency Regulation: Experimental Results
}

\author{
Philip J. Douglass Student Member, IEEE, Rodrigo Garcia-Valle Member, IEEE, Preben Nyeng \\ Member, IEEE, Jacob Østergaard Senior Member, IEEE, and Mikael Togeby
}

\begin{abstract}
As renewable energy sources increase their penetration, the traditional providers of frequency regulation service, fossil fueled thermal power plants, will be displaced, motivating the search for novel providers such as demandside resources. This paper presents the results of field experiments using demand as a frequency controlled reserve (DFCR) on appliances with programmable thermostats. The experiments conducted showed the response of a population of thermostatically controlled loads acting as normal reserves and disturbance reserves as defined by the Nordic Grid Codes [1]. In addition, industrial pump loads and relay-controlled loads were tested as DFCR.

The tests show that a population of refrigerators was able to deliver frequency reserves approximately equal to their average power consumption. Electric space heaters were able to provide frequency reserves of over $90 \%$ their maximum power consumption in certain weather conditions.
\end{abstract}

Index Terms-Demand side, frequency control, demonstration project.

\section{INTRODUCTION}

T RADITIONALLY, electric generators are dispatched to follow passive loads. This mode of operation is infeasible with non-dispatchable stochastic energy sources such as wind and PV and one possible remedy is to dispatch loads to follow production. Today, many residential loads are equipped with microprocessors running firmware for controlling local processes. These loads could be programmed to actively monitor the state of the power system as a whole and schedule their own power consumption to contribute to the stable and efficient operation of the system.

Loads providing thermal energy services (e.g. refrigerators, heat pumps and resistive heaters) are well suited to following fluctuating generation because their inherent heat capacity acts as an energy storage device allowing electricity consumption to be shifted in time without compromising the quality of service. Thermostat controlled loads (TCLs) are a significant portion of total electric loads, representing around half of household electricity consumption in the USA [2].

P. Douglass, R. Garcia-Valle, and J. Østergaard are with Centre for Electrical Technology, Technical University of Denmark, Elektrovej-Building 325, 2800 Kgs. Lyngby, Denmark \{pjdo, rgv, joe\}@elektro.dtu.dk.

Preben Nyeng is with Energinet.dk, Tonne Kjærsvej 65, 7000 Fredericia, Denmark pny@energinet.dk.

M.Togeby is with EA Energy Analyses, Frederiksholms Kanal 4, 3. th., 1220 Copenhagen K. mt@eaea.dk
Despite the declining cost of communications devices, providing a real-time digital communications interface from a system operator to small loads represents a significant cost barrier to widespread deployment. However, there is already a parameter which is universally available to indicate the instantaneous balance of electric energy production and consumption, namely the system frequency.

System operators seek to minimize the extent and duration of frequency deviations from the nominal value. The Nordic power system has been experiencing declining frequency quality for the past 10 years, in 2011 system frequency was outside the acceptable range of 50 $\mathrm{Hz} \pm 100 \mathrm{mHz}$ for more than $2 \%$ of the time [3]. During periods when frequency was below the acceptable range, insufficient frequency controlled reserves were available to satisfy the $n-1$ reliability criteria.

The relation between power generated, $P_{m}(t)$, power consumed, $P_{L}(t)$, and deviations in system frequency, $\Delta f(t)$, is given by

$$
\Delta P_{m}(t)-\Delta P_{L}(t)=2 H \frac{d \Delta f(t)}{d t}+D \Delta f(t)
$$

[4]

where $\mathrm{H}$ is the inertia constant, and $\mathrm{D}$ is the load damping coefficient.

Loads may measure the system frequency and by adjusting their power consumption up or down as the system frequency rises or falls, they are able to provide reserves for frequency regulation. This concept is known as demand as a frequency controlled reserve (DFCR) [5], or alternatively Frequency Adaptive Power Energy Rescheduler (FAPER) [6], Dynamic Demand [7], Frequency-Sensitive Gridfriendly ${ }^{\mathrm{TM}}$ Appliances [8], or Frequency Responsive Load Controller [9].

This paper presents the result of a field experiment where, for the first time, DFCR loads have been installed in an uncontrolled working environment and their performance as a group has been monitored.

The load damping coefficient captures the behavior of motors, which constitute a large portion of total load. DFCR loads' power use in aggregate is also proportional to system frequency, but there are several aspects that cause DFCR loads to be poorly modeled by their contribution to the load damping coefficient. These aspects are:

1) Time Dependency: DFCR loads imply an energy 
storage buffer, and this buffer's "state of charge" (SOC) depends on the historical progression of the system's frequency. The appliance's frequency response depends on the SOC the energy storage buffer.

2) Discrete nature of loads: many types of loads are either ON/OFF, it is only in aggregate that they can provide a gradual, linear frequency response.

3) Parameter Design: The damping coefficient of traditional loads is a natural property, rather than a design decision. With DFCR loads, the system planner has the freedom to specify the frequency response, rather than be constrained by the inherent properties of passive loads. The frequency response can be specified over at limited range of frequencies and be flat outside that band.

While the DFCR loads are physically located in the low voltage distribution system, it is the transmission system operator who needs to account for their behavior when specifying the requirements for frequency regulation reserves.

This paper is structured as follows: Section II describes the experimental setup including the design of the DFCR controller and loads, Section III describes the parameter configuration for operation in the Nordic power system. Section IV presents and discusses the results of the experiment. Finally, Section V concludes with a description of future work.

\section{EXPERIMENTAL SETUP}

We have currently deployed approximately 70 DFCR appliances out of a planned 200 units, primarily on an island in the Baltic Sea, Bornholm, which is connected to the Nordic transmission grid by a $60 \mathrm{kV}$ under-sea cable. Bornholm has a peak load of $55 \mathrm{MW}$ and a high penetration of wind energy (over $30 \%$ of electric energy production annually), but when the island is disconnected from the Nordic grid, wind production must be curtailed to maintain acceptable frequency quality [10], [11].

The DFCR systems consist of two parts: a commercially available appliance which has been modified to expose a serial port to an external controller, and an external controller which we have produced for this experiment from off-the-shelf components [12]. The TCLs are composed of bottle coolers located in hotels, restaurants and convenience stores, and resistive electric heating systems placed in single family homes.

\section{A. DFCR Controller Hardware}

Fig. 1 shows a block diagram of the DFCR controller. The DFCR controller measures frequency using a zerocrossing algorithm and averaging over 8 cycles. Every 250 $\mathrm{ms}$ the CPU receives and processes frequency measurements. The controller timestamps all measurements with a real-time clock that is synchronized via the internet time

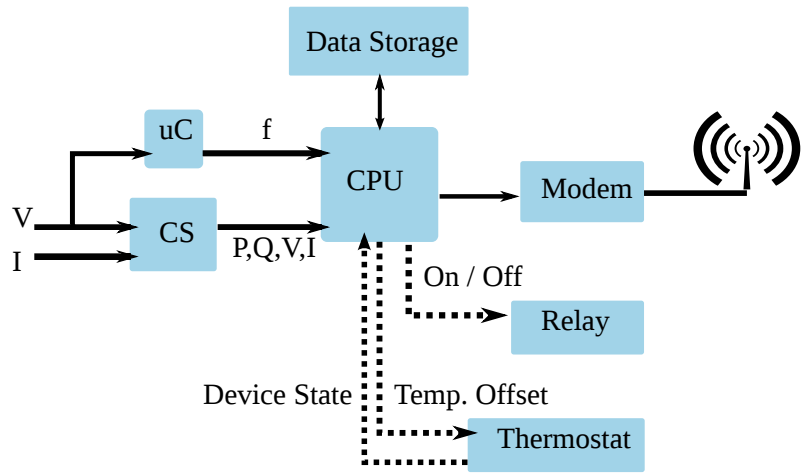

Figure 1. DFCR block diagram. The CPU is a low cost microcontroller with $8 \mathrm{kB}$ of RAM. The system frequency is measured by a secondary micro-controller (uC). Power consumption (real and reactive) is calculated by a dedicated component (CS). The measurements are buffered to an SD card (Data Storage), and uploaded periodically to our server via GSM/GPRS (Modem). Some boxes have a relay built into the device, others communicate to programmable thermostats via a serial cable.

protocol NTP. When multiple controllers take frequency measurements within the same second, the standard deviation of frequency measurements are $1.3 \mathrm{mHz}$ [12].

An integrated circuit dedicated to power measurement measures volage and current, and calculates active and reactive power consumption of the attached loads. Data on power consumption and system frequency, as well as parameters specific to the appliance under control are sampled once per minute, and stored into a large internal memory. In addition, when a large frequency excursion occurs, data is collected at a high resolution (as often as every 2 seconds). This data is periodically uploaded to a database using a GSM/GPRS wireless modem and the HTTP protocol.

The DFCR controller parameters are configurable, and the firmware can be remotely upgraded. This facility was used to test different types of frequency reserves.

\section{B. Loads}

1) Bottle Cooling Refrigerators: The refrigerators used in the experiment are bottle coolers with a glass door and internal light that remains on when the door is closed. They contained a programmable thermostat that, via a serial cable, delivered data to the controller about the internal state of the device and accepted configuration commands. The DFCR controller utilized a mode of the thermostat that added a temperature offset to the user-given setpoint. Only the operation of the compressor is affected by the external controller, the light and other internal processes which account for a residual power consumption are not affected by the DFCR function. Comparing power consumption before and while the compressor runs reveals that the compressor itself consumes on average $230 \mathrm{~W}$. When the compressor is off but the light is on the refrigerator consumes $30 \mathrm{~W}$ and when the light is off it consumes 13 W. The daily load profile of refrigerators, shown in fig. 2, 


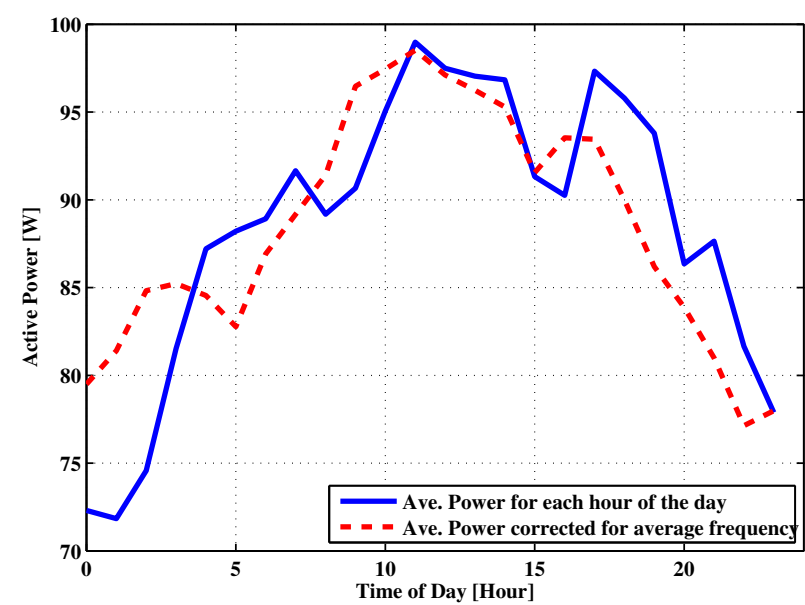

Figure 2. Power Consumption of the refrigerators for each hour of the day while operating as DFCR in normal reserve mode (solid blue line). Because the average frequency also varies through the hours of the day, removing the effect of average frequency variations gives an estimate of the load profile without DFCR functionality enabled (dashed red line).

reveals that the power consumption is higher in the day when users are actively using the appliances.

The user configures the refrigerator thermostat with a temperature setpoint. The thermostat turns the compressor on when the internal air temperature rises above the deadband of $2{ }^{\circ} \mathrm{C}$, and turns the compressor off when the air temperature reaches the setpoint. The thermostat includes an "anti-short cycle" feature, which ensures that at least 3 minutes elapse between stopping and restarting the compressor. This feature protects the motor from over loading due to high pressures in the condensor. During normal operation, without introducing setpoint offsets, the ON/OFF cycle repeats every 15 minutes, where the compressor has a duty cycle of $32 \%$.

The normal operation of the thermostat is periodically interrupted by the defrost cycle which turns the compressor off for approximately 30 minutes and allows the internal air temperature to rise well above the deadband. A refrigerator is in the defrost state $6 \%$ of the time. To analyze the effect of DFCR functionality, refrigerators in defrost state are excluded from the data set. The "anti-short cycle" feature also interferes with the ideal operation of the refrigerators, but unlike with the defrost state, there was no feedback from the thermostat to the DFCR controller as to when this feature was active, so its effect could not be explicitly accounted for.

In total, 40 refrigerators were deployed, and data was available from 26 of them for the time period chosen for analysis. The refrigerators that did not deliver data failed because of problems such as poor GSM connectivity or a missing serial connection between the controller and thermostat.

2) Electric Space Heaters: The electric heaters used in the experiment are resistive radiators in private residences with a rated power consumption of $490 \mathrm{~W}$. As with the

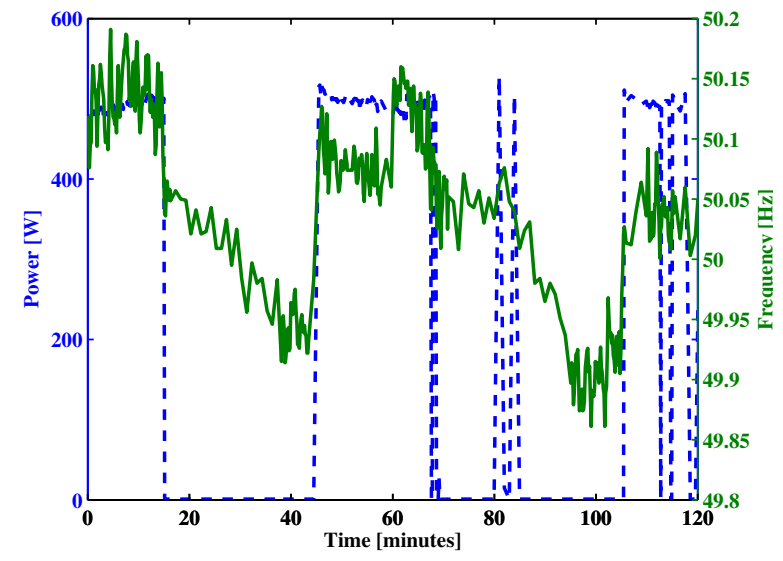

Figure 3. Time series of electric heating with DFCR controller active. System frequency is shown in solid green, power consumption from one heater is shown in dashed blue. Adjusting the heater's thermostat causes the heater to remain off when the frequency is below $50 \mathrm{~Hz}$ in this case.

refrigerators, the user gives a temperature setpoint, and the DFCR controller adds an offset to the setpoint depending on the system frequency. The operation of the thermostat is not as straightforward as the refrigerators because temperature measurements are filtered before being compared to the setpoint and deadband. This filtering is done to compensate for the heat generated by the microelectronics in the thermostat itself, and optimize power consumption while accounting for the heat capacity of a home and the behavior of its occupants.

Data from a typical 2 hour period is shown in fig. 3.

3) General Relay-Controlled Loads: Data was collected from 10 controllers equipped with a relay that deenergized attached loads. These units opened the relay when system frequency fell below a given configurable threshold, and reconnected when system frequency returned above a higher threshold, subject to time constraints on the minimum and maximum allowable disconnect time. Another time constraint ensured that after being disconnected, the load remained reconnected for a minimum time span. A more detailed presentation of this algorithm can be found in [5].

The loads connected to this controller were diverse including pumps for circulating water, resistive heaters, and small refrigerators. These loads were located in educational institutions, offices and homes.

4) Wastewater Treatment Plant: Treatment of wastewater is an energy intensive service with a large untapped potential for demand response. In Denmark wastewater treatment consumed $528 \mathrm{GWh}$ of electric energy in 2009, accounting for $1.6 \%$ of all electricity consumption [13]. The central wastewater treatment plant serving Bornholm participated in the DFCR experiment by allowing some non-critical loads to be controlled to provide frequency controlled disturbance reserves. These loads were in the form of induction motors that pumped water, and moved 
cleaning brushes. The DFCR control box provided a binary input into an existing industrial control system which was responsible for actuating the loads. A signal from a DFCR controller indicated when the system frequency had fallen below a given threshold, and the industrial control system was reprogrammed to use this signal to interrupt processes that tolerated interruption, while giving first priority to ensuring that process constraints were not violated. The behavior of these loads are comparable to the relay-controlled loads, with the exception that the time constraints are handled by the industrial control system, not the DFCR controller.

DFCR control units were attached to each of the controlled loads acting exclusively as power measurement devices. Data from 13 loads representing an average power consumption of $115 \mathrm{~W}$ was analyzed. Two $50 \mathrm{~kW}$ pumps were also controlled based on the DFCR signal, representing more load than the remaining population of DFCR loads combined, but data from these pumps was unavailable at the time of writing because the current transformer of the DFCR controller was too small to give accurate measurements.

\section{CONFIGURATIONS OF DFCR FOR THE NORDiC SYSTEM}

The Nordic grid maintains frequency stability by purchasing frequency controlled reserves from the market in 4 hour blocks one day in advance. In the hour of operation, the system operator monitors system frequency for off-nominal excursions and tie lines for deviations from scheduled transfers, and manually activates the least cost up or down regulation resources to correct any imbalances. In the event of an imbalance between power supply and demand, the frequency controlled reserves act to stop the system frequency from changing, but they do not restore the frequency to the nominal value. At present, the Nordic system lacks an automatic frequency restoration reserve, and this results in long periods when the system frequency operates at off-nominal values.

The frequency controlled reserves are divided into two subcategories: Normal Reserve and Disturbance Reserve. The normal reserve is active in the range $49.90 \mathrm{~Hz}-$ $50.10 \mathrm{~Hz}$, and requires a linear response from generators within $180 \mathrm{~s}$. Generators which participate in this reserve are continuously adjusting their output to match the small fluctuations in system frequency, but their slow response, while favorable to operators of thermal power plants, has a negative effect on frequency quality. The disturbance reserve is active in the range $49.50 \mathrm{~Hz}-49.90 \mathrm{~Hz}$. It is also a linear response, but it must act faster than the normal reserve, being 50\% activated within $5 \mathrm{~s}$, and fully activated within $30 \mathrm{~s}$ [1]. This type of reserve is intended to act on rare occasions, such as when a transmission line, or power plant trips. At present, the disturbance reserve is activated about once an hour.
Table I

PARAMETERS FOR NORMAL RESERVE

\begin{tabular}{|l|l|l|}
\hline Controller Type & Parameter Name & Value \\
\hline TCL & Minimum Temperature Offset & $-2^{\circ} \mathrm{C}$ \\
TCL & Maximum Temperature Offset & $2^{\circ} \mathrm{C}$ \\
TCL & Lower Frequency Response Limit & $49.90 \mathrm{~Hz}$ \\
TCL & Upper Frequency Response Limit & $50.10 \mathrm{~Hz}$ \\
\hline
\end{tabular}

\section{A. DFCR for Normal Reserve}

The TCLs are well suited for continuous operation as a normal reserve, because the setpoint offsets can be effectively done in $0.1^{\circ} \mathrm{C}$ increments. When the devices were configured to operate as a normal reserve, the user given temperature setpoint corresponded to the thermostat setting at the nominal system frequency, $50 \mathrm{~Hz}$. The thermostat temperature setpoint was offset from the user given setpoint by a value linearly proportional to the deviation of the system frequency from nominal as described in [14].

The range of setpoint variations was chosen to exceed the size of the thermostat's deadband, so that a sudden change from $50 \mathrm{~Hz}$ to $49.90 \mathrm{~Hz}$ would turn all devices off, including those that had recently turned on. Values for the controller's parameters are given in table I. The relay-controlled loads, and the loads of the wastewater treatment plant are not suitable for operating continuously as a normal reserve.

\section{B. DFCR for Disturbance Reserve}

For the TCLs, operation as a disturbance reserve is similar to the normal reserve, with the difference being that the setpoint offset first takes effect at $-100 \mathrm{mHz}$ from nominal frequency. The temperature offset range of the TCLs operating as disturbance reserve is $+3^{\circ} \mathrm{C}$ at $49.70 \mathrm{~Hz}$ and $0^{\circ} \mathrm{C}$ at $49.90 \mathrm{~Hz}$. This is a smaller range, but a larger deviation of temperature from the user given setpoint than the normal reserve, a choice which is justified by the short time periods spend in this frequency range.

The relay-controlled loads, and the loads of the water treatment plant were all programmed to shed load at 49.90 Hz. Using a single cutoff threshold simplified the implementation and analysis of the devices, but from a system's perspective this is undesirable behavior. The risk caused by this implementation is exemplified by the large cohort of PV inverters in Germany which are all programmed to cut off production at $50.20 \mathrm{~Hz}$ [15]. In a large scale deployment, the threshold frequency would need to be spread over a range of values to avoid disturbances caused by step changes in load.

The relay-controlled loads were given conservative time constraints to accommodate the diversity of load types. Refrigerators represented the most sensitive loads: the maximum disconnect time must be short enough not to degrade food quality, minimum disconnect time must be long enough to allow the pressure in the condenser to equalize, and the minimum reconnect time must be long 
Table II

PARAMETERS For Disturbance RESERVE

\begin{tabular}{|l|l|l|}
\hline Controller Type & Parameter Name & Value \\
\hline TCL & Minimum Temperature Offset & $-3^{\circ} \mathrm{C}$ \\
TCL & Maximum Temperature Offset & $0^{\circ} \mathrm{C}$ \\
TCL & Lower Frequency Response Limit & $49.70 \mathrm{~Hz}$ \\
TCL & Upper Frequency Response Limit & $49.90 \mathrm{~Hz}$ \\
Relay & Minimum Disconnect Time & $30 \mathrm{~s}$ \\
Relay & Maximum Disconnect Time & $120 \mathrm{~s}$ \\
Relay & Minimum Reconnect Time & $240 \mathrm{~s}$ \\
Relay/Water & Cutoff Frequency & $49.90 \mathrm{~Hz}$ \\
Relay/Water & Reconnect Frequency & $49.95 \mathrm{~Hz}$ \\
\hline
\end{tabular}

enough to allow the internal air temperature to recover from being disconnected. These time constraints were fixed, so they must account for the worst case situation. This resulted in time constrains shown in table II.

\section{RESULTS AND DISCUSSION}

This section presents the results of the experiment, grouped by configuration type and load type.

\section{A. Normal Reserve}

1) Refrigerators: Data was taken from 26 refrigerators over 16 weeks. Samples of frequency, power and temperature were taken by each control box every minute. The samples were sorted chronologically and the mean frequency, power consumption, and temperature values were found for each minute. The resulting data set for each minute in the 16 week period had less variation compared to the samples from each control box. The data from each minute was grouped by system frequency value and then the mean power consumption and temperature was found for each frequency group. The results for power consumption, shown in fig. 4 , are well fit by a linear least squares approximation. The data set is less dense at frequency extremes because system frequency follows a Gaussian distribution around $50.00 \mathrm{~Hz}$. At frequencies above $50.10 \mathrm{~Hz}$ and below $49.90 \mathrm{~Hz}$, the linear trend breaks down because the thermostat's offset has reached the limit of it's deviation from the user-given setpoint.

The slope of the least squares linear regression is $431 \mathrm{~W} / \mathrm{Hz}$. Given that the thermostat was changed with $20{ }^{\circ} \mathrm{C} / \mathrm{Hz}$, the relation of temperature offset to power consumption is $21.6 \mathrm{~W} /{ }^{\circ} \mathrm{C}$. The difference in average power consumption at $50.10 \mathrm{~Hz}$ and $49.90 \mathrm{~Hz}$ was 132 $\mathrm{W}-41.9 \mathrm{~W}=90.1 \mathrm{~W}$. Compared to the compressor's power consumption of $230 \mathrm{~W}$, we find that $39.2 \%$ of the compressor's power has been mobilized to participate in DFCR service. The average power consumption of the refrigerators (including light and residual consumption) was $89.4 \mathrm{~W}$, slightly less than the power provided for the frequency response.

The distribution of values within each frequency group was analyzed by finding the quartiles, as shown in fig. 5 . The difference between quartiles increases as frequency

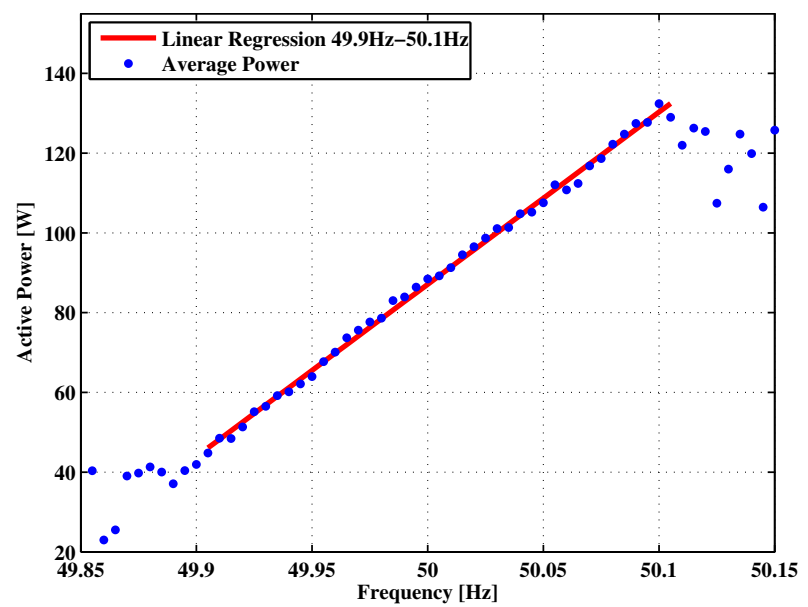

Figure 4. Frequency Response of refrigerators with least squares linear regression. Temperature offset varied linearly with $\pm 2^{\circ} \mathrm{C}$ in the range $49.90 \mathrm{~Hz}-50.10 \mathrm{~Hz}$, with $0^{\circ} \mathrm{C}$ offset at $50.00 \mathrm{~Hz}$.

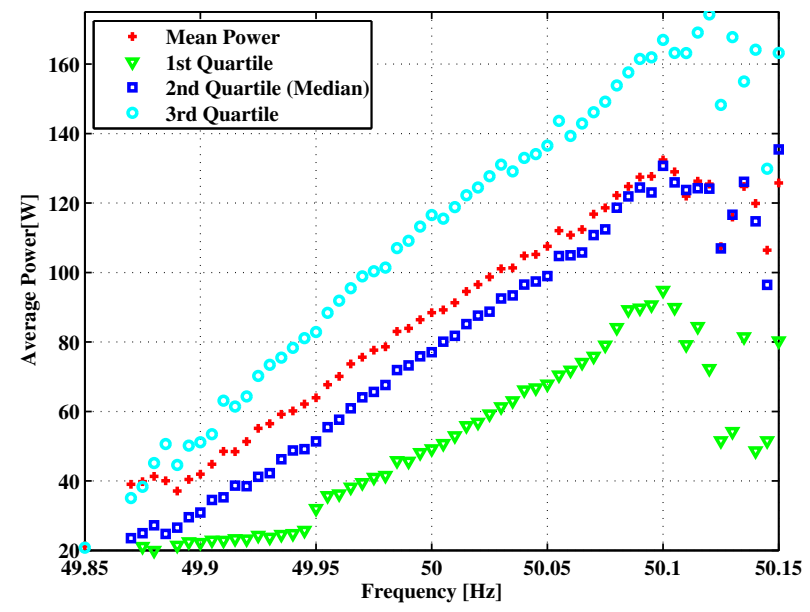

Figure 5. For each frequency group, mean power is shown together with the median, 1st and 3rd quartiles.

increases. For frequencies below $49.95 \mathrm{~Hz}$, the first quartile is where all compressors in the population are off.

The power consumed by the refrigerators' compressor is used to cool the air inside, but the air temperature changes more slowly than power consumption, and is delayed by the heat capacity of the heat transfer circuit. Plotting average internal air temperature against average frequency for each minute, fig. 6 shows an inverse correlation of temperature to system frequency, as expected. The average temperature varies by approximately $\pm 1^{\circ} \mathrm{C}$ from $49.90 \mathrm{~Hz}$ - $50.10 \mathrm{~Hz}$, even though the thermostat setpoint has been offset by $2^{\circ} \mathrm{C}$.

Continuously changing the refrigerators setpoint offset increased the number of times that the compressor cycled ON and OFF by $10 \%$ compared to non-DFCR operation.

To reveal how the frequency response changed due to the frequency history, the data was divided into 3 groups based on the average historical frequency: low historical frequency $(\bar{f}<49.975 \mathrm{~Hz})$, middle historical frequency 


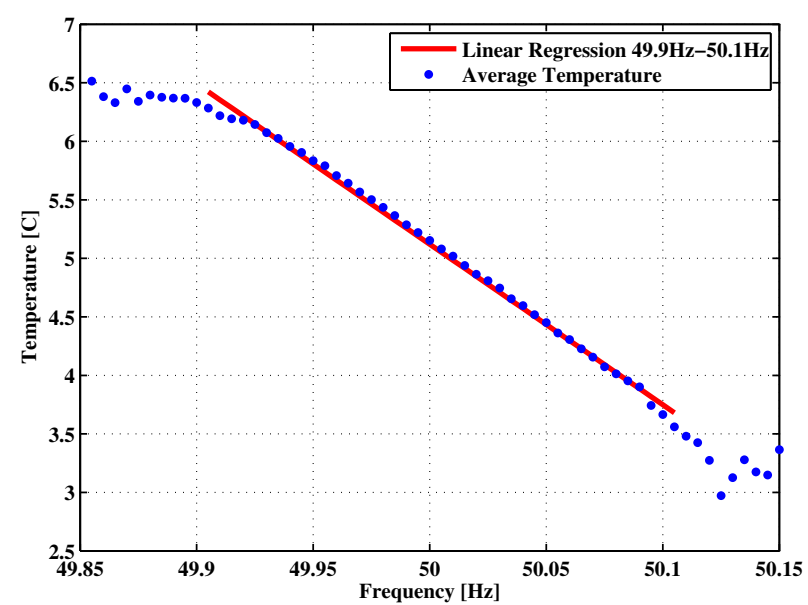

Figure 6. Average internal air temperature of refrigerators vs frequency with least squares linear regression.

(49.975 $\mathrm{Hz}<\bar{f}<50.025 \mathrm{~Hz})$ and high historical frequency $(\bar{f}>50.025 \mathrm{~Hz})$. The frequency thresholds dividing groups were chosen to balance the number of samples falling into each group, with $50 \%$ of samples in the middle group. Comparing the frequency response of the 3 groups shows how it is influenced by the progression of frequency in the recent past. When the historical frequency has been high, the average power consumed at nominal frequency is lower than when the historical frequency has been in the middle or low range. This is because the internal temperatures of the refrigerators have been lowered by offsetting the thermostats' setpoints. When the historical frequency is high, returning to nominal frequency draws on the stored thermal energy, and reduces the average power consumption. The converse happens when the historical frequency is lower than nominal. Figure 7 shows the frequency response of the 3 groups when averaging the historical frequency over 6 minutes. The time period for averaging frequency values was varied from 2 to 20 minutes to reveal that time scale which has the most impact on the frequency response. The difference between the 3 groups is quantified by finding a linear best fit of each group, and then comparing the expected values at nominal frequency. The difference in expected values, shown in fig. 8 , rises to a peak at 6 minutes before declining gradually. This indicates that the heat capacity of the refrigerators is able to shift power consumption for 6 minutes, after which time, the ability to shift power further in time is reduced.

2) Electric Heaters: The quantity of data from the electric heaters was not as extensive as for the refrigerators because of their small number, and weather dependence. The data collected from 2 houses over 11 days at a one minute sampling interval was grouped according to frequency in shown in fig. 9. The linear response is clearly visible until $49.93 \mathrm{~Hz}$. At this frequency value, the power consumption reaches $6 \mathrm{~W}$ and can not be reduced further.

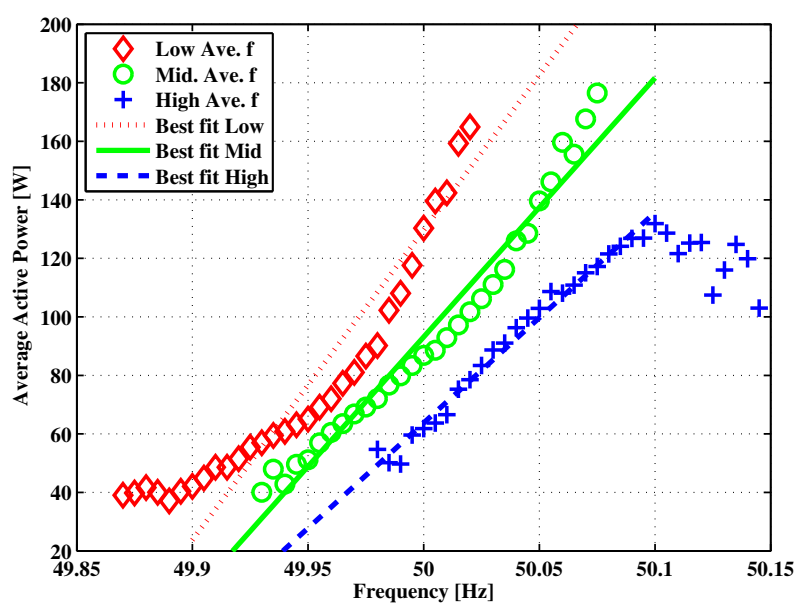

Figure 7. Frequency response at low, middle and high historical frequencies when calculating average frequency over 6 minutes, with best fit lines.

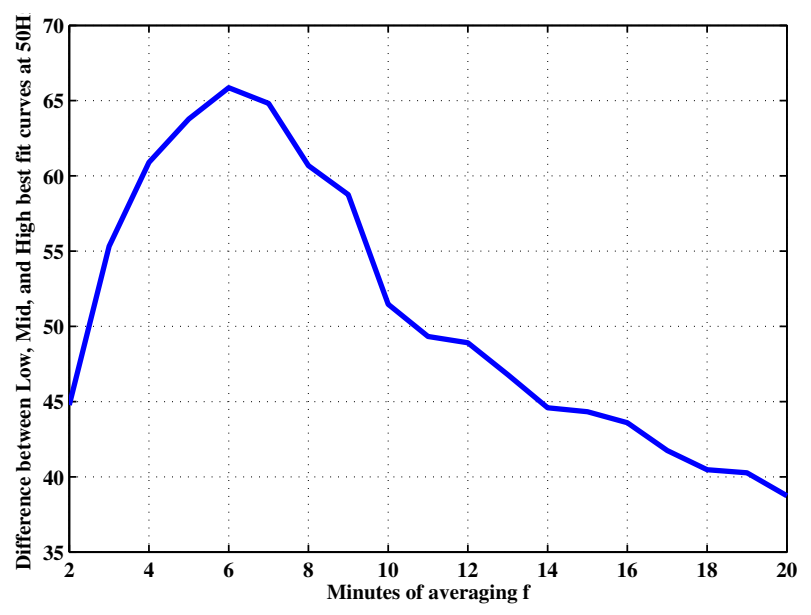

Figure 8. The difference between low, middle and high frequency best fit lines at nominal frequency for different sizes of time windows for calculating average frequency.

At $50.10 \mathrm{~Hz}$, the average power consumption of $456 \mathrm{~W}$, giving a frequency response of $450 \mathrm{~W}$, equivalent to $92 \%$ of the maximum power consumption of the heaters. The slope of the best fit line is $2.83 \mathrm{KW} / \mathrm{Hz}$. The average power consumption was $185 \mathrm{~W}$, so the frequency response was $240 \%$ of the average power. These metrics will depend greatly on the ambient temperature, and the time period under consideration represents favorable conditions.

\section{B. Disturbance Reserve}

1) Refrigerators: The refrigerators were reconfigured to operate as a disturbance reserve for a two week period. Analyzing the samples shown in fig. 10 shows that, despite the noise caused by a relatively small data set at extreme values, a frequency response is apparent at frequency values below $49.90 \mathrm{~Hz}$ and frequencies above this value gave no response. The slope of the best fit line in the range $49.80 \mathrm{~Hz}-49.90 \mathrm{~Hz}$ is $595 \mathrm{~W} / \mathrm{Hz}$, or $40 \mathrm{~W} /{ }^{\circ} \mathrm{C}$. 


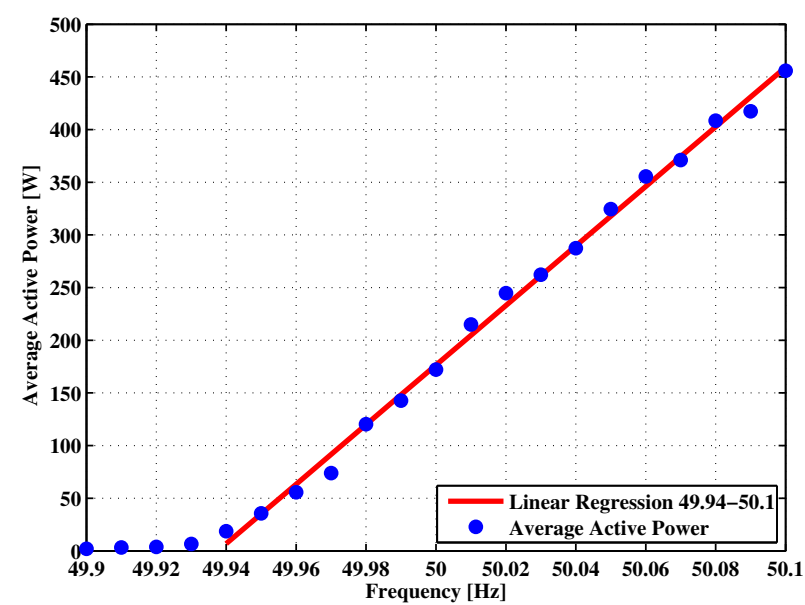

Figure 9. Frequency response of electric heaters with least squares linear regression. The average power consumption approached $0 \mathrm{~W}$ before the thermostat's offset limit at $49.90 \mathrm{~Hz}$ was reached.

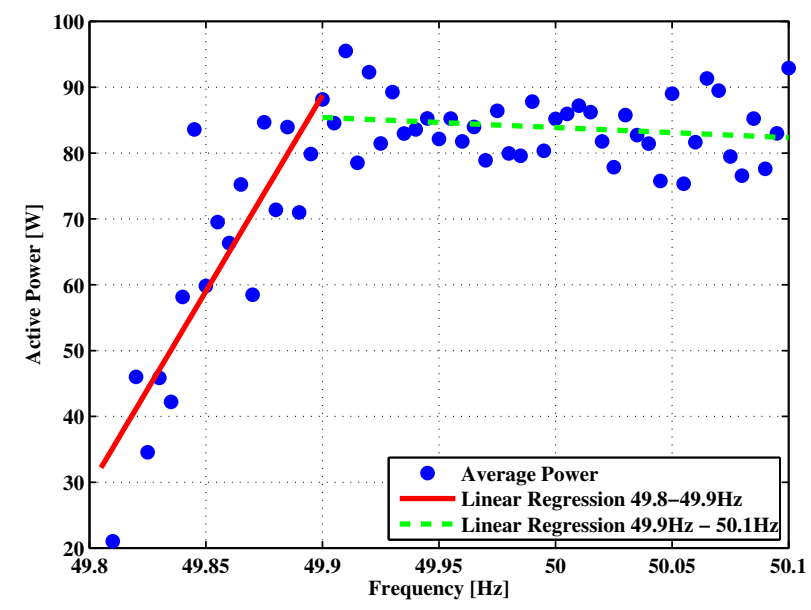

Figure 10. Frequency response of refrigerators acting as a disturbance reserve, shown with piecewise least squares linear regression in regions above and below $49.90 \mathrm{~Hz}$.

Despite the fact that the slope of the temperature offset as a disturbance reserve $\left(15^{\circ} \mathrm{C} / \mathrm{Hz}\right)$ is lower than in the normal reserve $\left(20^{\circ} \mathrm{C} / \mathrm{Hz}\right)$, the frequency response per degree of temperature offset is almost twice as much. An explanation of this behavior can be found by considering that when a disturbance occurs the internal temperatures of the refrigerators are most likely in the nominal state, giving large room for deferring power consumption for the short duration of extreme under-frequency events. In the normal reserve case, system frequency is seen to dwell at offnominal values for extended periods of time, weakening the average response.

The size of the data set at extreme frequencies is too small to conclude the total amount of frequency response provided by the refrigerators. Where a reasonable quantity of measurements exist at $49.82 \mathrm{~Hz}$ the refrigerator used an average of $46 \mathrm{~W}$, indicating at this frequency the response was $37.1 \mathrm{~W}$, equivalent to $44 \%$ of the average power, and

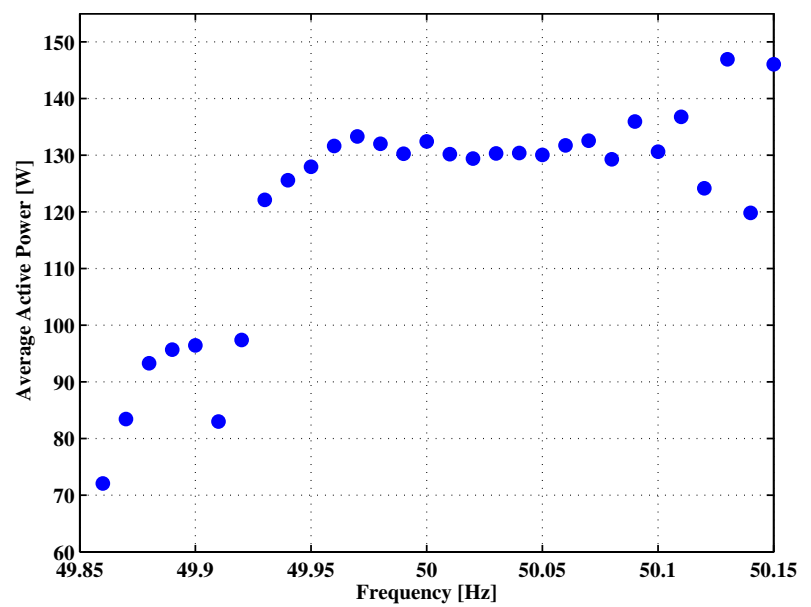

Figure 11. Frequency response of water treatment plant configured with disconnect frequency $49.90 \mathrm{~Hz}$ and reconnect frequency $49.50 \mathrm{~Hz}$.

$16 \%$ of the compressor's power.

2) Water Treatment Plant: The frequency response of the water treatment plant is shown in fig. 11. Figure 11 shows something approximating a step change in power consumption around the cutoff frequency, which is blurred by the time constraints (min. and max. disconnect times) applied to the loads. The loads used on average $132 \mathrm{~W}$ at frequencies above the reconnect frequency and $83.5 \mathrm{~W}$ at frequencies below the cutoff value, giving a frequency response equivalent to $36.7 \%$ of the power consumption above the reconnect frequency.

3) Relay-Controlled Loads: During the experimental period, the frequency response of the relay-controlled was the opposite of what we intended: lower frequencies corresponded to higher power consumption. This is explained by the dominating influence of time constraints on the state of the relays. When the system frequency was below the cutoff value, $60 \%$ of the time the loads were energized because of the constraint on the maximum OFF time and minimum $\mathrm{ON}$ time after reconnection. When frequency was above the cutoff value, $1 \%$ of the time the relays had de-energized loads because of the minimum disconnection time constraint. The peak in power consumption occurs at the reconnect frequency, $49.95 \mathrm{~Hz}$, and this is because of an inrush current and rebound effect as the loads restore their desired state after being interrupted. But the maximum OFF time constraint meant that the loads could be energized at other low frequency values, and this is reflected in the results showing power consumption at all low frequencies values higher that when operating at nominal frequency.

The controller algorithm itself is not invalidated by these results, it is parameter values need to be revised. The implementation behaved as specified, the problem was that the time constraints were not tuned to the actual frequency conditions of the Nordic power system. This power system experiences numerous frequency excursions below 49.90 $\mathrm{Hz}$ which are sustained for a duration longer than the 
maximum time the loads are allowed to be disconnected. Raising the reconnect frequency would help mitigate the problem associated with the minimum reconnect time, and to work around the maximum disconnect time constraint the cutoff frequency could be lowered, so the reserve is active less often and for shorter time periods. Appliances with more relaxed time constraints are more likely to provide the desired response at higher cutoff frequency values.

\section{CONCLUSion AND Future Work}

The work with DFCR appliances presented in this paper builds on previous laboratory experiments by scaling up the number of frequency controlled devices, increasing the diversity of loads under control, and testing them during daily use.

In absolute terms, the amount of power under DFCR control in this experiment was rather modest, a few kilowatts at most. However, relative to the power demand of each of the TCLs, the frequency response was significant. For demand-side resources in the residential sector to become economically viable, the fixed costs of providing this functionality must be small to match the small power demand of each individual unit. The DFCR controllers used in this experiment were not themselves cost effective, but the use of low-cost components for the core functions of measuring frequency and executing the DFCR algorithm justify optimism that when designing appliances, the marginal costs of providing this feature approach negligible levels.

An analysis of the frequency and consumption data found that while operating as a frequency reserve in the range $49.90 \mathrm{~Hz}-50.10 \mathrm{~Hz}, 39.2 \%$ of the refrigerators' compressor power could be utilized for frequency regulation, and $92 \%$ of the home heaters' power could be utilized during the time period studied. The loads under control in the wastewater treatment plant reduced power consumption by $36.7 \%$ during under-frequency events. The response of relay-controlled loads were sensitive to the time constraints, frequency threshold values and the distribution of frequency values for synchronous system where they are connected. The slope of response measured as $\mathrm{W} / \mathrm{Hz}$ was larger when the refrigerators operated as a disturbance reserve, though the magnitude of response was smaller.

Data collection continues and new loads, including more home heating systems and two large pumps, will be added to the population of appliances under control. In the future we will perform a time domain analysis to characterize the speed of frequency response.

Finally, because the DFCR controllers allow all control algorithms to be remotely upgraded, the experimental platform is generally useful for other demand response studies. When the DFCR study in concluded, the controllers will be reprogrammed to respond to an external price signal, rather than system frequency.

\section{ACKNOWLEDGEMENTS}

This work was funded by Danish Energy Agency's "Energy Technology Development and Demonstration Program" (EUDP). Special thanks to Christian Brandt Rasmussen, Rune Brus, Nina Marie Holmboe and Janos Hethey for their contributions.

\section{REFERENCES}

[1] Nordel, "System operation agreement," Online https://www.entsoe.eu/, 13 Jun. 2006, accessed April 27, 2012. [Online]. Available: https://www.entsoe.eu/

[2] Energy Information Administration, "Residential Energy Consumption Survey," U.S. Dept. Energy, Washington, DC, Tech. Rep., 2009.

[3] E-Bridge, "Analysis \& Review of Requirements for Automatic Reserves in the Nordic Synchronous System," Tech. Rep., 21 May 2011.

[4] Hassan Bevrani, Robust Power System Frequency Control. Springer, 2009.

[5] Zhao Xu, J. Østergaard, and M. Togeby, "Demand as frequency controlled reserve," Power Systems, IEEE Transactions on, vol. 26, no. 3, pp. $1062-1071$, aug. 2011.

[6] F. Schweppe, R. Tabors, J. Kirtley, H. Outhred, F. Pickel, and A. Cox, "Homeostatic utility control," Power Apparatus and Systems, IEEE Transactions on, vol. PAS-99, no. 3, pp. 1151 -1163, may 1980.

[7] J. Short, D. Infield, and L. Freris, "Stabilization of grid frequency through dynamic demand control," Power Systems, IEEE Transactions on, vol. 22, no. 3, pp. $1284-1293$, aug. 2007.

[8] N. Lu and D. Hammerstrom, "Design considerations for frequency responsive grid friendly(tm) appliances," in Transmission and Distribution Conference and Exhibition, 2005/2006 IEEE PES, May 2006, pp. $647-652$.

[9] A. Molina-Garcianda, F. Bouffard, and D. Kirschen, "Decentralized demand-side contribution to primary frequency control," Power Systems, IEEE Transactions on, vol. 26, no. 1, pp. $411-419$, feb. 2011

[10] Y. Chen, Z. Xu, and J. Ostergaard, "Frequency analysis for planned islanding operation in the danish distribution system - bornholm," in Universities Power Engineering Conference, 2008. UPEC 2008 43rd International, sept. 2008, pp. $1-5$.

[11] G. Tarnowski, P. Kjær, J. Østergaard, and P. Sørensen, "Frequency control in power systems with high wind power penetration," in 9th International Workshop on Large-Scale Integration of Wind Power into Power Systems, 2010.

[12] P. Douglass, R. Garcia-Valle, P. Nyeng, J. Østergaard, and M. Togeby, "Demand as Frequency Controlled Reserve: Implementation and practical demonstration," Innovative Smart Grid Technologies Europe, 2011.

[13] Danish Energy Association, "Dansk Elforsyning Statistik," 2009.

[14] P. Nyeng, J. Østergaard, M. Togeby, and J. Hethey, "Design and implementation of frequency-responsive thermostat control," in Universities Power Engineering Conference (UPEC), 2010 45th International, 31 2010-sept. 3 2010, pp. 1 -6.

[15] K. Burges, P. Zolotarev, and J. Lehner, "Impact of Large-scale Distributed Generation on Network Stability During OverFrequency Events \& Development of Mitigation Measures," Sep. 2011. [Online]. Available: http://www.vde.com/en/fnn/pages/50-2hz-study.aspx 